\title{
Mise au point d'une suspension bactérienne épaissie pour l'exobiocicatrisation de matériaux cimentaires fissurés
}

\author{
I. Feurgard ${ }^{1,2,3, a}$, C. Lors $^{2,3}$, R. Gagné ${ }^{1}$ et D. Damidot ${ }^{2,3}$
}

Reçu le 14 juin 2016, accepté le 3 septembre 2016

\begin{abstract}
Résumé - La précipitation de carbonate de calcium par les bactéries offre la possibilité de réparer les matériaux cimentaires fissurés par une méthode durable et non polluante. Une suspension bactérienne épaissie a été mise au point pour favoriser la biocicatrisation de microfissures entre 150 et $500 \mu \mathrm{m}$ en utilisant la souche bactérienne Bacillus pseudofirmus. Le fluide obtenu, par synergie entre deux épaississants colloïdaux, est fortement rhéofluidifiant et thixotrope, résistant à la décantation et forme lors d'un repos prolongé un gel physique réversible. Ces propriétés permettent de l'injecter sans drainage post-injection dans des fissures d'ouverture variant de 150 à $500 \mu \mathrm{m}$. L'ajout d'épaississants n'a pas d'impact sur la croissance ni sur l'activité de B. pseudofirmus et entraîne la formation d'amas autour des bactéries. Cette structure similaire à un biofilm favorise potentiellement leur survie face aux contraintes du milieu extérieur.
\end{abstract}

Mots clés : Mortier / microfissures / bactéries / biominéralisation / épaississant

\begin{abstract}
Bacterial precipitation of calcium carbonate opens the way to a non-polluting and long lasting method for the remediation of cracked cementitious materials. We characterized a thickened bacterial suspension for its ability to be injected in fine cracks (150 to $500 \mu \mathrm{m})$ and to promote biocicatrisation by Bacillus pseudofirmus. The suspension, due to a synergy between two colloidal thickeners, is a strongly shear-thinning and thixotropic fluid, is resistant to settling, and can form a weak gel at rest. These properties allow its injection without drainage in 150 to $500 \mu \mathrm{m}$ wide cracks. The thickened medium does not alter B. pseudofirmus growth or activity, and the bacteria are embedded in hutches creating a biofilm-like structure. This structure may provide a protective environment for the bacteria, enhancing their survival and activity in hostile conditions.
\end{abstract}

Key words: Mortar / micro-sized cracks / bacteria / biomineralization / thickeners

\section{Introduction}

La formation de microfissures dans les ouvrages en matériaux cimentaires permet l'entrée de composés agressifs au cour du matériau et accélère leur vieillissement. Le colmatage des microfissures est actuellement réalisé à l'aide de résines synthétiques. Toutefois, ces composés sont toxiques et polluants, et nécessitent un renouvellement régulier du traitement [1-3]; ce qui augmente le coût et l'impact environnemental générés par ces réparations. L'agent de réparation du futur pourrait être la calcite biogénérée, un matériau durable et naturel. En effet, certaines bactéries peuvent produire par biominéralisation des carbonates de calcium solides à partir de calcium dissous [4]. Réalisée dans les fissures, la biominéralisation peut offrir un mode de colmatage intéressant : la biocicatrisation [5-7].

Si la plupart des travaux visent à introduire les bactéries dans le matériau pour le rendre auto-réparant (autobiocicatrisation), il est aussi possible d'injecter les bactéries dans des fissures comme un agent de réparation (exobiocicatrisation). Assurer l'activité bactérienne dans les fissures des matériaux cimentaires reste cependant un défi important : ce sont des environnements hostiles et exposés aux agressions physiques, chimiques et biologiques du milieu extérieur. En outre, les cultures

\footnotetext{
a Auteur pour correspondance : ivan.feurgard@mines-douai.fr

1 Centre de recherche sur les infrastructures en béton (CRIB), Université de Sherbrooke, 2500 Boulevard de l'Université, J1K2R1 Sherbrooke, Québec, Canada

2 Mines Douai, LGCgE-GCE, 941 rue Charles Bourseul, 59508 Douai, France

3 Université Lille Nord de France, Lille, France
} 
bactériennes en milieu liquide ne permettent pas le maintien de l'ensemble bactéries-nutriments-eau dans les fissures. L'enjeu est donc de concevoir un milieu non conventionnel pouvant être injecté aisément dans des microfissures de plus de $150 \mu \mathrm{m}$, résistant à l'écoulement, et permettant aux bactéries de produire du carbonate de calcium en quantités suffisantes. Plusieurs méthodes d'immobilisation bactérienne ont été employées, telles que la mousse polyuréthane [8], des billes de verre [9] ou du sable $[10,11]$. Toutefois, elles ne possèdent pas toutes les propriétés décrites précédemment. Le Silicagel [12-14] et l'Agar [15] sont des vecteurs prometteurs, mais leur gélification rapide rend leur mise en œuvre délicate.

Les épaississants colloïdaux naturels ont un fort potentiel pour la conception d'un milieu injectable pour l'exobiocicatrisation de microfissures. En effet, ils permettent d'immobiliser simplement les bactéries, sont faciles à préparer, non polluants et biocompatibles [16]. De plus, ces additifs sont pour certains fortement rhéofluidifiants et/ou thixotropes [17]; leur viscosité peut donc être aisément contrôlée pour réaliser une injection efficace et limiter l'écoulement post-injection. Enfin, ces composés forment dans certains cas des structures protectrices autour des bactéries, procurant des avantages similaires à ceux d'un biofilm : protection contre la prédation par les protozoaires, résistance à la dessiccation, résistance au lessivage des bactéries et des nutriments [18].

Cette étude vise à caractériser les propriétés rhéologiques d'une suspension bactérienne comprenant deux épaississants colloïdaux, ainsi que son impact sur la bioprécipitation de carbonate de calcium par Bacillus pseudofirmus, dans l'optique de mettre en place une méthodologie de réparation de microfissures d'ouverture comprise entre 150 et $500 \mu \mathrm{m}$.

\section{Matériel et Méthodes}

\subsection{Essais rhéologiques}

Les suspensions étudiées sont caractérisées à l'aide d'un rhéomètre à cylindres coaxiaux (MCR302, Anton Parr) équipé d'un cylindre rotatif cannelé (modèle CC27). Pour les caractérisations simples, la contrainte de cisaillement est mesurée pour des taux de cisaillement décroissants de $100 \mathrm{~s}^{-1}$ à $0,1 \mathrm{~s}^{-1}$ après une pré-agitation à $100 \mathrm{~s}^{-1}$ durant $50 \mathrm{~s}$. Pour caractériser la thixotropie, la contrainte de cisaillement est mesurée sous un cisaillement négligeable durant 10 min après une pré-agitation à $500 \mathrm{~s}^{-1}$ durant $10 \mathrm{~s}$.

Les tests de gélation sont réalisés par ajout de $15 \mathrm{~mL}$ de suspension préalablement agitée dans un tube de $50 \mathrm{~mL}$. Le tube est retourné au bout d'un temps donné. La suspension est gélifiée si le fluide ne s'écoule pas lors $\mathrm{du}$ retournement.

Les essais sont réalisés avec le milieu mis au point par Ducasse-Lapeyrusse (2014) [21] contenant 75 g.L L $^{-1}$ de L-lactate de calcium hydraté (Sigma-Aldrich), 47 g.L $\mathrm{L}^{-1}$ de nitrate de calcium tétrahydraté et 3 g. $\mathrm{L}^{-1}$ d'extrait de levure (Difco), modifié par addition de 20 g.L ${ }^{-1}$ de
D-gluconate de calcium (Sigma-Aldrich). Il est épaissi à l'aide d'un biopolymère $\mathrm{A}$ et d'une argile $\mathrm{B}$ par dispersion à froid des poudres dans le milieu. Les concentrations de $\mathrm{A}$ et $\mathrm{B}$ sont égales respectivement à $0,4 \%$ pour $\mathrm{A}$ et $6 \%$ pour B (pourcentage massique d'épaississant par rapport à la masse totale des constituants du milieu). Le $\mathrm{pH}$ initial du milieu épaissi est de 6,1 contre 6,5 pour le milieu non épaissi. Les suspensions contenant uniquement A ou B ont également été testées pour comparer leur rhéologie à celle du mélange.

Un fluide Newtonien se définit par une viscosité constante, un fluide rhéofluidifiant a une viscosité réduite lorsqu'il est soumis à un cisaillement, et un fluide thixotrope devient progressivement moins visqueux lorsqu'il est soumis à un cisaillement, de manière réversible une fois au repos.

\subsection{Essais d'injection}

Les essais d'injection sont réalisés sur des éprouvettes de mortier fissurées, préparées selon la procédure décrite par Ducasse-Lapeyrusse et al. [19] : les mortiers utilisés pour ces essais ont été préparés d'après la norme ASTM $\mathrm{C} 109 \mathrm{M}$, avec un ratio eau/ciment de 0,485 et un ratio sable/ciment de 2,75, en utilisant du sable normalisé d'Ottawa et un ciment Portland ordinaire (GU) canadien ayant une finesse Blaine de $381 \mathrm{~m}^{3} \cdot \mathrm{kg}^{-1}$. Les mortiers ont ensuite été curés pendant 28 jours à $23{ }^{\circ} \mathrm{C}$ et à $100 \%$ $\mathrm{RH}$. Des fissures réalistes d'ouverture comprise entre 150 et $500 \mu \mathrm{m}$ sont réalisées par forçage d'un cœur expansif dans l'éprouvette selon la procédure décrite par Gagné et Argouges (2012) [20], puis sont carbonatées pendant un mois à $20{ }^{\circ} \mathrm{C}$, à $4 \%$ de $\mathrm{CO}_{2}$ et $40 \% \mathrm{HR}$, afin de simuler le vieillissement naturel des fissures, réduire leur $\mathrm{pH}$ et supprimer le biais lié à la formation d'une couche d'autocicatrisation naturelle. L'ouverture des fissures est mesurée à l'aide d'un vidéomicroscope en 24 points répartis sur les faces inférieure et supérieure de l'éprouvette. Les essais d'injection sont réalisés par injection de $10 \mathrm{~mL}$ de suspension à l'aide d'une seringue de $20 \mathrm{~mL}$, au moyen d'un sabot d'injection spécialement adapté, les bords de la fissure étant colmatés à l'aide d'adhésif pour éviter les fuites (Fig. 1).

La suspension est injectée progressivement dans la microfissure, le dispositif d'injection est ensuite retiré et l'excédent est arasé. La suspension est trop épaisse si l'injection ne permet pas un remplissage total de la fissure ou impose une trop forte pression, et trop fluide si un drainage du fluide de la fissure est constaté après injection. Le Methocel J 20 MS, épaississant stable et aisé à préparer, est utilisé comme référence. L'efficacité de l'injection est estimée en ajoutant 1 g. $\mathrm{L}^{-1}$ de bleu de méthylène dans la suspension, afin de visualiser les zones recouvertes par le milieu. Après $48 \mathrm{~h}$ de séchage à température ambiante, la fissure est ouverte pour observer les deux faces intérieures de la fissure.

Afin d'observer de façon plus précise le comportement de la suspension dans une fissure, une fissure de $400 \mu \mathrm{m}$ 

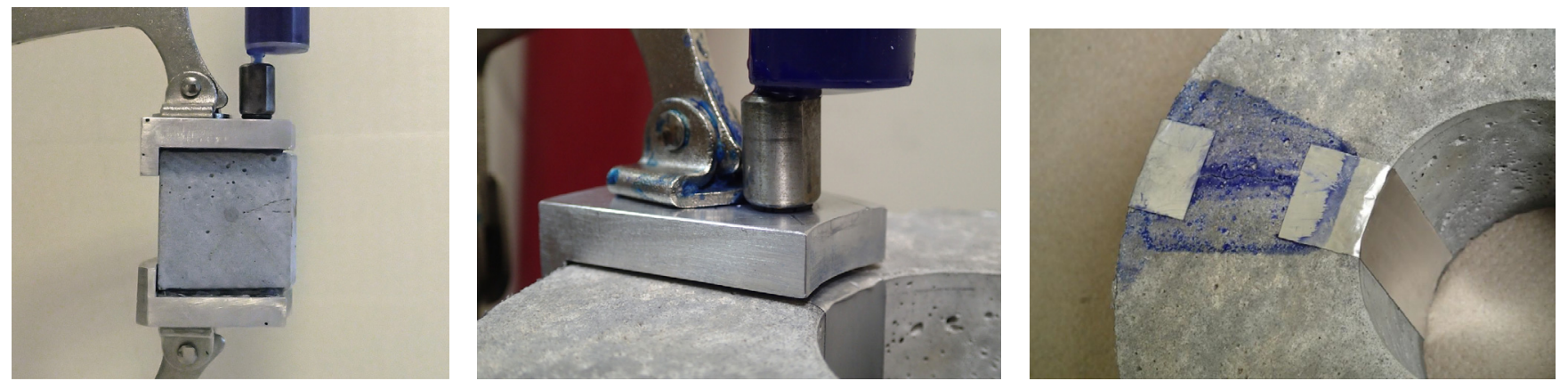

Fig. 1. De gauche à droite - Vue en coupe du dispositif d'injection placé sur une éprouvette de mortier; vue de la partie supérieure; éprouvette fissurée après injection d'une solution colorée.

Fig. 1. From left to right - cross-sectional view of the injection device setup on a mortar sample; upper side view; cracked mortar sample after injection of a coloured solution.

d'ouverture est simulée en maintenant deux cubes de mortier de 5 cm de côté séparés par deux lames d'inox. L'injection est réalisée de la même manière que pour une fissure réelle. Après $48 \mathrm{~h}$ de séchage à température ambiante, $60 \mathrm{~mL}$ d'eau sont injectés, puis après $48 \mathrm{~h}$ de séchage à température ambiante, les deux cubes sont séparés à température ambiante pour observer l'intérieur de la fissure simulée.

\subsection{Essais de croissance bactérienne}

Les bactéries (Bacillus pseudofirmus, DSMZ) sont précultivées durant $40 \mathrm{~h}$ dans un milieu contenant 8 g.L $\mathrm{L}^{-1}$ de bouillon nutritif (Nutrient Broth, Difco) et 11,8 g.L ${ }^{-1}$ de nitrate de calcium tétrahydraté (Sigma-Aldrich) à $30{ }^{\circ} \mathrm{C}$ et placé sur une table rotative à $150 \mathrm{rpm}$. Le $\mathrm{pH}$ initial du milieu de pré-culture est de 7,6.

Les essais de biominéralisation sont réalisés en triplicat dans le milieu de culture décrit précédemment dans la Section 2.1, inoculé avec $100 \mu \mathrm{L}$ de préculture dans des erlenmeyers de $250 \mathrm{~mL}$ contenant $100 \mathrm{~mL}$ de milieu et mis en culture à $20^{\circ} \mathrm{C}$ sur une table rotative à $150 \mathrm{rpm}$. Le milieu de culture est stérilisé par filtration sous vide à $0,2 \mu \mathrm{m}$.

Les essais en milieu épaissi sont réalisés à $20{ }^{\circ} \mathrm{C}$ dans du milieu contenant les deux épaississants A et B aux concentrations de $0,4 \%$ et $6 \%$ respectivement. Les épaississants sont stérilisés par autoclave et dispersés à froid dans le milieu.

La croissance cellulaire est suivie par comptage direct de neuf champs d'observation à l'aide d'un microscope à épifluorescence (Leica DMLP) après coloration de la suspension bactérienne par un fluorochrome (acridine orange). Les concentrations en lactate et en gluconate sont déterminées par dosage du lactate par chromatographie ionique (DIONEX ICS-3000). En fin d'essai, la culture est centrifugée à $8000 \mathrm{rpm}$ durant $20 \mathrm{~min}$ à $4{ }^{\circ} \mathrm{C}$. Le culot est ensuite rincé avec $20 \mathrm{~mL}$ d'eau milli-Q pour $50 \mathrm{~mL}$ de culture (deux rinçages pour les milieux épaissis) et séché à $40{ }^{\circ} \mathrm{C}$ durant $48 \mathrm{~h}$ pour déterminer la teneur en matière sèche de la culture. La quantité de carbonate

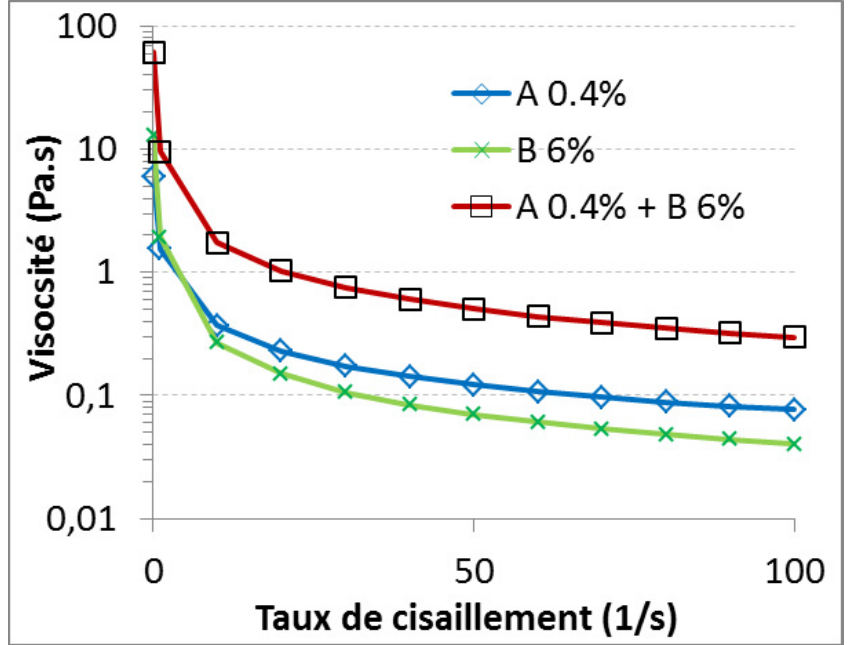

Fig. 2. Rhéogrammes des suspensions contenant les épaississants A et B individuellement et en mélange.

Fig. 2. Rheograms of suspensions containing thickeners $A$ and $B$, separately and mixed.

de calcium formé est quantifiée par analyse thermogravimétrique (ATG, NETZCH STA449F3).

\section{Résultats}

\subsection{Caractérisation rhéologique des suspensions épaissies}

Une caractérisation des suspensions contenant un seul épaississant a été dans un premier temps réalisée. Les suspensions contenant les épaississants A $(0,4 \%)$ ou B $(6 \%)$ sont fortement rhéofluidifiantes, mais présentent toutefois des comportements rhéologiques différents (Fig. 2) :

- L'épaississant A a un pouvoir épaississant fortement supérieur à l'épaississant B : à $100 \mathrm{~s}^{-1}$, une suspension à $0,4 \%$ de $\mathrm{A}$ a une viscosité de $7,7 \times 10^{-2} \mathrm{~Pa}$ contre $4,0 \times 10^{-2} \mathrm{~Pa}$ pour une suspension à $6 \%$ de $\mathrm{B}$; 
- L'épaississant B est plus rhéofluidifiant, propriété recherchée pour le contrôle de la viscosité : pour les mêmes concentrations que précédemment, à faible cisaillement $\left(0,1 \mathrm{~s}^{-1}\right)$ la suspension $B$ est plus épaisse (12,9 Pa.s contre 5,9 Pa.s pour la suspension A);

- L'épaississant B est thixotrope, conservant temporairement une viscosité réduite après avoir été soumis à un cisaillement, propriété absente pour A dont la viscosité est stable dans le temps à cisaillement constant ;

- Les suspensions B décantent en quelques heures, tandis que les suspensions A sont stables et sont toujours homogènes au bout de 10 jours de repos.

Suite à ces essais, une suspension utilisant les deux épaississants A et B en mélange a été testée, afin d'exploiter les synergies existant entre eux. Ces synergies permettent de mettre au point une suspension dotée des propriétés rhéologiques recherchées pour cette étude : stabilité, pouvoir épaississant, caractère rhéofluidifiant et caractère thixotrope.

La suspension épaissie à l'aide des épaississants A et $\mathrm{B}$ (à la concentration de $0,4 \%$ et $6 \%$ respectivement) forme un fluide épais homogène, qui ne montre aucune ségrégation ou décantation après 10 jours de repos. Séchée à l'air libre, la suspension forme un film solide, qui ne se dissout pas au mouillage mais devient souple. Les autres constituants du milieu n'ont pas d'impact direct sur ses propriétés rhéologiques. Caractérisée au rhéomètre, la suspension épaissie montre un fort comportement rhéofluidifiant et thixotropique ainsi qu'une importante augmentation de viscosité par rapport aux suspensions ne contenant qu'un seul épaississant présent dans les mêmes proportions (Fig. 2).

Elle est également thixotrope, ce qui se traduit par un regain progressif de la viscosité au repos (Fig. 3). Elle peut en outre former un gel physique (pseudo-gel) après plusieurs heures de repos. Ces gels peuvent être déstructurés à volonté par agitation et se restructurent au repos.

Ces observations révèlent une synergie entre les deux épaississants, ayant les effets suivants sur la rhéologie :

- Stabilisation de la suspension contre la décantation et la ségrégation;

- Augmentation de la viscosité;

- Comportement rhéofluidifiant;

- Comportement thixotrope.

Les propriétés rhéologiques mesurées d'une suspension à $0,4 \%$ de $\mathrm{A}$ et $6 \%$ de $\mathrm{B}$ ont été comparées au modèle d'Ostwald pour des fluides pseudo-plastiques : son comportement est similaire à un pseudo-plastique d'Ostwald pour un indice de consistance $K=10,05$ et un indice de comportement $n=0,235$.

\subsubsection{Essais d'injection}

La suspension épaissie à l'aide des deux épaississants a une large plage d'utilisation. En effet, les suspensions ayant $0,4 \%$ de $\mathrm{A}$ et $6 \%$ de $\mathrm{B}$ ont pu être injectées aisément, sans drainage post-injection et avec un taux de

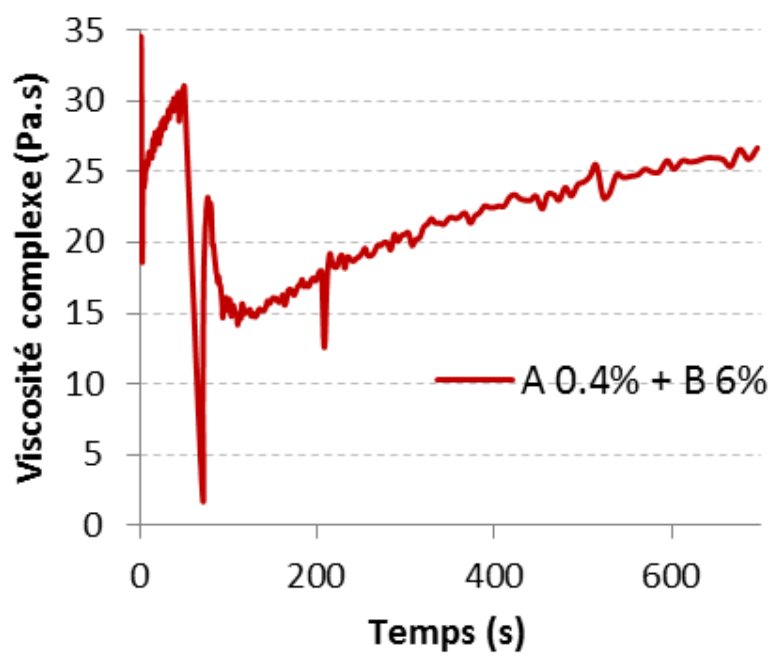

Fig. 3. Rhéogramme de thixotropie de la suspension contenant les deux épaississants.

Fig. 3. Thixotropy rheogram of a suspension containing both thickeners.

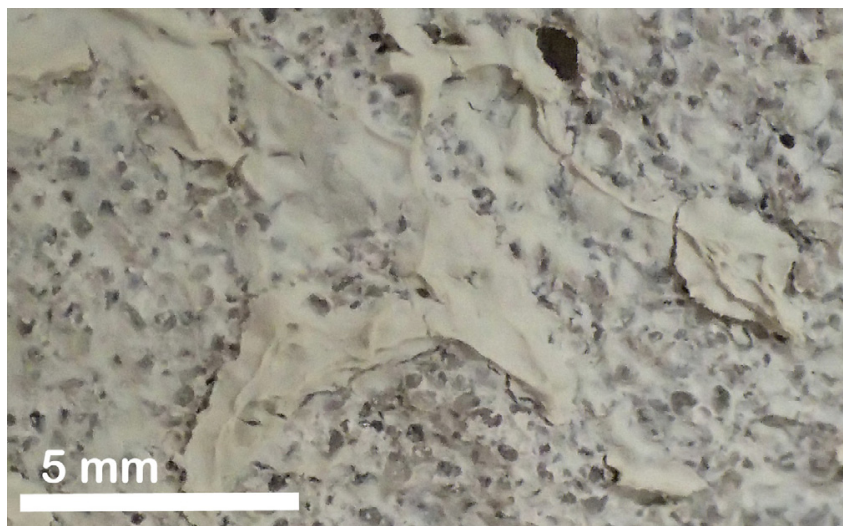

Fig. 4. Photographie du film formé à l'intérieur de la fissure d'un mortier après injection puis séchage de la suspension épaissie.

Fig. 4. Photography of the film formed inside a crack of a mortar by the thickened suspension after injection and drying.

remplissage supérieur à $80 \%$ dans des fissures de 150 à $500 \mu \mathrm{m}$. Cette polyvalence est directement liée à la combinaison entre effet rhéofluidifiant et thixotrope : la forte baisse de viscosité engendrée par l'agitation préalable de la suspension épaissie permet l'injection dans des fissures fines, mais le regain de viscosité est suffisamment rapide et important pour assurer le maintien de la suspension dans la fissure une fois l'injection terminée. En revanche, la suspension témoin à base de Methocel, peu rhéofluidifiante et non thixotrope, a une plage d'utilisation d'environ $150 \mu \mathrm{m}$ avec une tendance prononcée au drainage. Après injection de la suspension épaissie puis séchage du mortier pendant $48 \mathrm{~h}$ à $20^{\circ} \mathrm{C}$, on observe à l'intérieur de la fissure du mortier un dépôt formé par la suspension sèche, formant un film ou une croûte selon la largeur de la fissure (Fig. 4). 
A

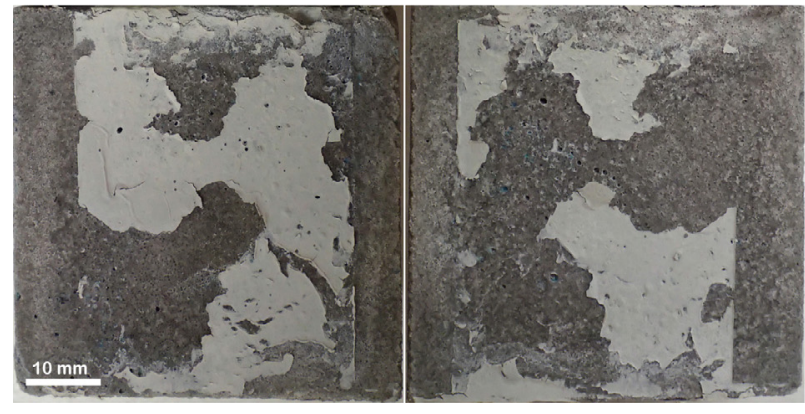

B

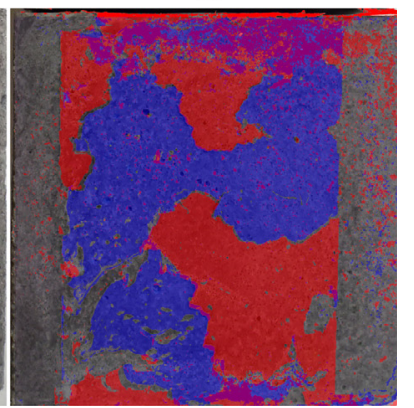

Fig. 5. A - Photographies des faces intérieures d'une fissure simulée de $400 \mu \mathrm{m}$ après injection de suspension puis d'eau distillée. B - superposition informatique des deux faces intérieures de la fissure.

Fig. 5. A - Photography of the internal faces of a simulated crack after injection of thickened medium, followed by distilled water. B - Simulated superposition of both faces of the crack.
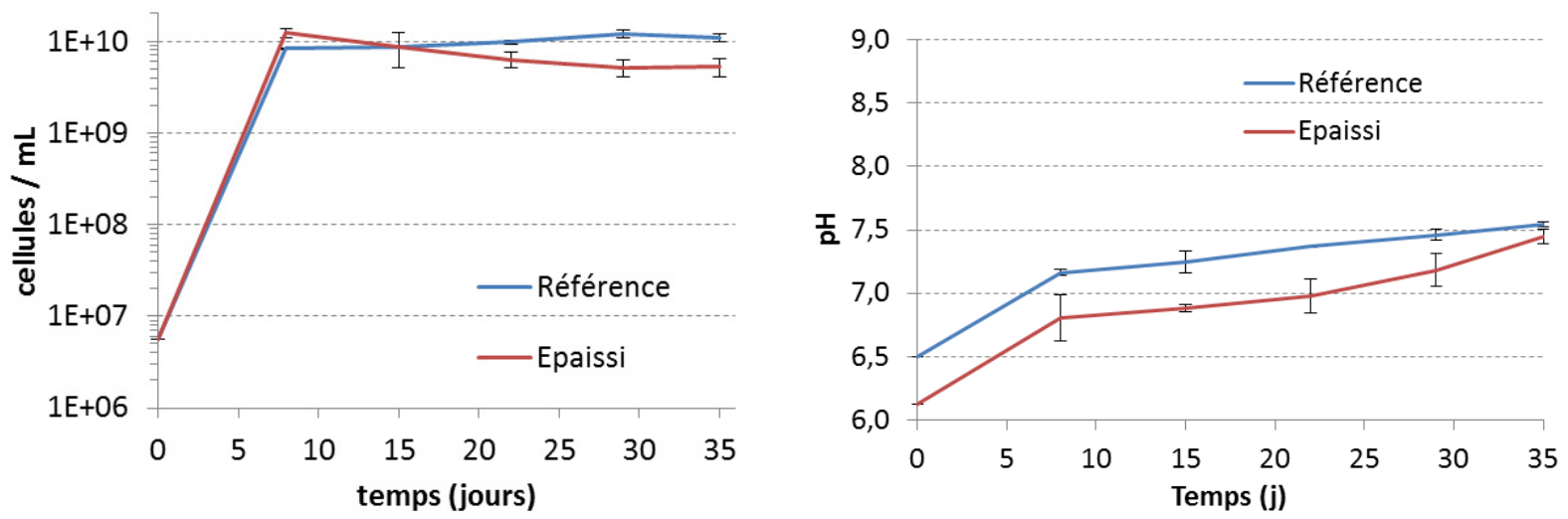

Fig. 6. Suivi de la croissance bactérienne et du pH de la culture de Bacillus pseudofirmus en milieux non épaissi et épaissi. Fig. 6. Bacterial growth and $p H$ of a batch of Bacillus pseudofirmus in non-thickened and thickened cultivation media.

Pour la fissure simulée (400 $\mu \mathrm{m}$ d'ouverture), l'injection de $60 \mathrm{~mL}$ d'eau après injection et séchage de la suspension épaissie ne provoque aucun lessivage, l'eau de sortie étant limpide et dépourvue de particules solides. En outre, l'ouverture du dispositif après ce test montre que la quasi-totalité de la surface de la fissure simulée est encore recouverte par le film (Fig. 5). La formation de ce film présente donc un double avantage pour le colmatage des microfissures : (1) il augmente potentiellement la couche de biocicatrisation formée par les bactéries et augmente ainsi l'efficacité du procédé de réparation; (2) favorise le maintien des bactéries au sein de la microfissure en leur constituant un support d'immobilisation et de nutriments.

\subsection{Essais de croissance bactérienne}

En présence des deux milieux, la population bactérienne atteint une population maximale de $5 \times 10^{9}$ bactéries.mL $\mathrm{mL}^{-1}$ au bout de 5 jours d'incubation, qui se maintient durant les 35 jours de l'essai et s'accompagne d'une augmentation progressive du pH. Pour la suspension épaissie, une décroissance progressive de la population bactérienne est observée, sans impact sur la production de carbonate de calcium. Cette décroissance est probablement due au regroupement progressif des bactéries en clusters qui biaise leur dénombrement. L'ajout des épaississants A et B $(0,4 \%$ et $6 \%$ respectivement) n'a pas d'influence sur la cinétique de croissance ni sur la taille de la population bactérienne en phase stationnaire (Fig. 6).

Dans toutes les cultures, les bactéries se développent sous forme de cocci, en tétrades isolées ou regroupées en clusters. Ce changement de morphologie est probablement une réponse au stress dû à l'ajout de nitrate de calcium $[21,22]$. Dans la suspension épaissie, les bactéries (points clairs) sont contenues dans des amas d'aspect fibreux (zones grises) et sont très peu présentes hors de ces amas (Fig. 7). Ces structures, observées seulement dans la suspension épaissie, sont formées des épaississants colloïdaux et forment une matrice autour des cellules, ce qui pourrait favoriser la survie et l'activité bactériennes. En effet, des interactions entre bactéries et colloïdes (tels que les argiles) peuvent conduire à des effets positifs sur l'activité bactérienne [23, 24].

Après 35 jours d'incubation, la consommation de lactate est de $92 \%( \pm 8 \%)$ pour le milieu non épaissi et $96 \%( \pm 4 \%)$ pour le milieu épaissi (Fig. 8). En outre, une 


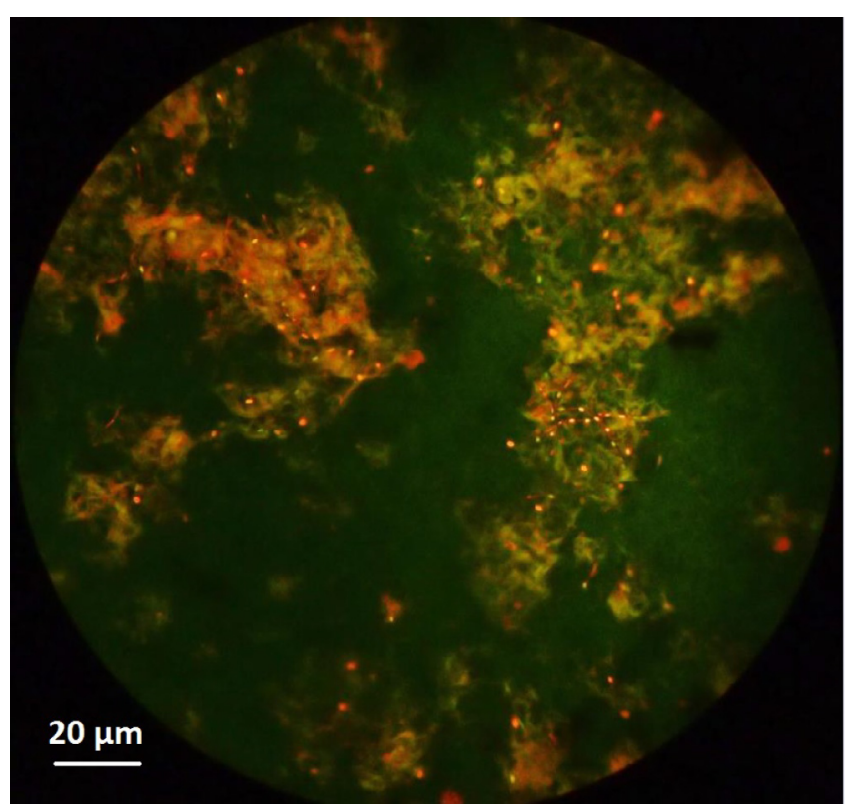

Fig. 7. Culture épaissie colorée à l'acridine orange observée au microscope à épifluorescence, après 8 jours d'incubation. Fig. 7. Epifluorescence micrograph of bacterial broth in thickened medium, after 8 days of cultivation.

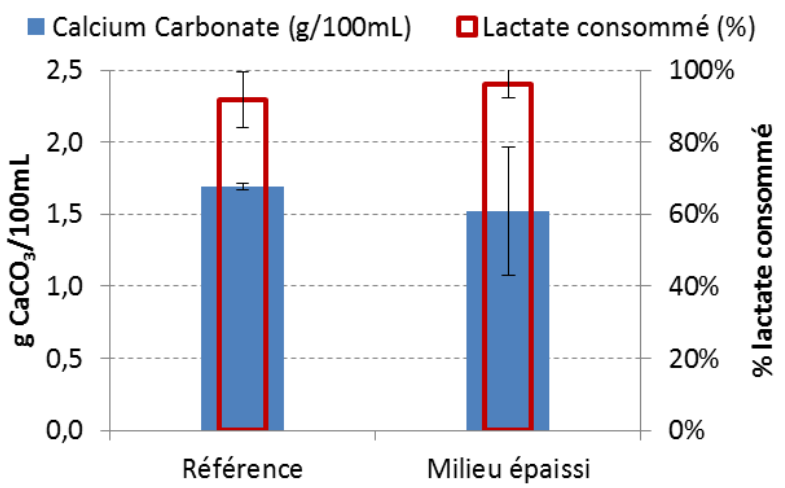

Fig. 8. Consommation de lactate et quantité de carbonate de calcium biogénéré après 35 jours de culture dans le milieu d'étude.

Fig. 8. Lactate uptake and biogenic calcium carbonate production after 35 days of cultivation in the studied medium.

diminution de la surface des pics du gluconate est observable pour les essais menés avec les deux milieux, mais n'est pas quantifiable du fait de pics parasites. Il y a donc eu dans les deux cas une consommation quasiment totale du lactate de calcium par les bactéries, engendrant la précipitation d'une quantité significative de carbonate de calcium. La quantité de carbonate de calcium biogénéré après 35 jours d'incubation est en effet de $1,7 \mathrm{~g}( \pm 0,02 \mathrm{~g})$ pour $100 \mathrm{~mL}$ de culture dans le milieu non épaissi. Elle est équivalente en milieu épaissi, avec une masse de 1,5 g $( \pm 0,45 \mathrm{~g})$ pour $100 \mathrm{~mL}$ de culture (Fig. 8). 30 à $40 \% \mathrm{du}$ calcium initialement présent dans le milieu sont convertis en carbonate de calcium, un taux de conversion proche de ceux obtenus par Ducasse-Lapeyrusse [25] et Ersan et al. [26]. L'ajout des épaississants A et B dans le milieu d'étude n'a donc pas d'effet sur la croissance et l'activité bactériennes, et permet la biominéralisation d'une quantité significative de carbonate de calcium.

\section{Conclusion}

L'utilisation d'un mélange d'épaississants collö̈daux a permis de formuler une suspension bactérienne épaissie aux propriétés particulièrement intéressantes :

- Préparation simple par dispersion à froid;

- Utilisation de réactifs peu coûteux et non polluants;

- Résistance à la décantation et stabilité dans le temps ;

- Comportement thixotrope et rhéofluidifiant permettant de former des gels physiques;

- Formation d'un film insoluble dans la fissure;

- Pas d'effets néfastes sur la croissance bactérienne.

La suspension a donc un fort potentiel pour réaliser l'exobiocicatrisation de microfissures de 150 à $500 \mu \mathrm{m}$ à l'échelle pilote, en facilitant non seulement le procédé d'injection des bactéries mais aussi en leur permettant de précipiter des quantités significatives de carbonate de calcium dans la microfissure. Simple à préparer et peu coûteuse, cette suspension peut être utilisable en conditions réelles. Enfin, la structure colloïdale de la suspension peut lui conférer des propriétés similaires aux biofilms, favorisant la survie et l'activité des bactéries dans la fissure. Au-delà de la biocicatrisation, ce type de suspension peut être employé dans des bioprocédés variés nécessitant l'immobilisation de bactéries sur des surfaces ou à l'intérieur d'espaces réduits.

\section{Références}

[1] F. Pacheco-Torgal, J.A. Labrincha, Const. Build. Mater. 40 (2013) 1136-1141

[2] M.C.S. Ribeiro, J.M.L. Reis, A.J.M. Ferreira, A.T. Marques, Polym. Test. 22 (2003) 849-857

[3] E.V. Sargent, C. Mitchell, R.E. Brubaker, Arch. Environm. Health : An Int. J. 31 (1976) 236-240

[4] C. Dupraz, R.P. Reid, O. Braissant, A.W. Decho, R.S. Norman, P.T. Visscher, Earth-Science Rev. 96 (2009) 141-162

[5] W. De Muynck, N. De Belie, W. Verstraete, Ecological Eng. 36 (2010) 118-136

[6] V. Achal, A. Mukherjee, D. Kumari, Q. Zhang, EarthScience Rev. 148 (2015) 1-17

[7] J. Ducasse-Lapeyrusse, C. Lors, R. Gagné, D. Damidot, Matériaux Techniques 103 (2015)

[8] S.S. Bang, J.K. Galinat, V. Ramakrishnan, Enzyme Microbial Technol. 28 (2001) 404-409

[9] S.S. Bang, J.J. Lippert, U. Yerra, S. Mulukutla, V. Ramakrishnan, Int. J. Smart Nano Mater. 1 (2010) 28-39

[10] S.K. Ramachandran, ACI Mater. J. (2001) 3-9

[11] S.K. Annamalai, K.D. Arunachalam, K.S.S. Sathyanarayanan, Mater. Res. Bull. 47 (2012) 3362-3368 
[12] K. Van Tittelboom, N. De Belie, W. De Muynck, W. Verstraete, Cem. Concr. Res. 40 (2010) 157-166

[13] V. Wiktor, H.M. Jonkers, Case Studies in Construction Materials (2015) 0-6

[14] N. De Belie, W. De Muynck, Concrete Repair, Rehabilitation Retrofitting II (2009) 777-782

[15] C. Qian, L. Ren, B. Xue, T. Cao, Const. Build. Mater. 106 (2016) 126-132

[16] S.C.S. Martins, C.M. Martins, S.T. Santaella, African J. Biotechnol. 2013 (2013) 4412-4418

[17] D.R. Picout, S.B. Ross-Murphy, The Scientific World Journal 3 (2003) 105-121

[18] K.C. Marshall, Ann. Rev. Phytopathol. 13 (1975) 357-373

[19] J. Ducasse-Lapeyrusse, R. Gagné, C. Lors, D. Damidot, Matériaux Techniques 102 (2014) 105

[20] R. Gagné, M. Argouges, Mater. Struct. 45 (2012) 16251638
[21] S.S. Justice, D. Hunstad, a, L. Cegelski, S.J. Hultgren, Nat. Rev. Microbiol. 6 (2008) 162-168

[22] K.D. Young, Curr. Opinion in Microbiol. 10 (2007) 596600

[23] H. Lünsdorf, R.W. Erb, W.R. Abraham, K.N. Timmis, Environm. Microbiol. 2 (2000) 161-168

[24] L.S. England, H. Lee, J.T. Trevors, Soil Biology Biochemistry 25 (1993) 525-531

[25] J. Ducasse-Lapeyrusse, Étude du potentiel d'autocicatrisation et de biocicatrisation de matériaux cimentaires fissurés, Thèse de Doctorat, Université Lille 1 et Université de Sherbrooke, École des Mines de Douai, 2014

[26] Y. Ç. Erşan, N. Belie, N. De, Boon, Biochem. Eng. J. 101 (2015) 108-118 